\title{
A Linguistic Multigranular Sensory Evaluation Model for Olive Oil
}

\author{
Luis Martínez, Macarena Espinilla, Luis G. Pérez \\ Dpt. of Computer Science, University of Jaen \\ 23071 - Jaen, Spain \\ E-mail: martin, mestevez,lgonzaga@ujaen.es
}

Received:19-01-2008

Revised:26-01-2008

\begin{abstract}
Evaluation is a process that analyzes elements in order to achieve different objectives such as quality inspection, marketing and other fields in industrial companies. This paper focuses on sensory evaluation where the evaluated items are assessed by a panel of experts according to the knowledge acquired via human senses. In these evaluation processes the information provided by the experts implies uncertainty, vagueness and imprecision. The use of the Fuzzy Linguistic Approach ${ }^{32}$ has provided successful results modelling such a type of information. In sensory evaluation it may happen that the panel of experts have more or less degree knowledge of about the evaluated items or indicators. So, it seems suitable that each expert could express their preferences in different linguistic term sets based on their own knowledge. In this paper, we present a sensory evaluation model that manages multigranular linguistic evaluation framework based on a decision analysis scheme. This model will be applied to the sensory evaluation process of Olive Oil.
\end{abstract}

\section{Introduction}

Evaluation is a complex cognitive process that involves different mechanisms in which it is necessary to define the elements to evaluate, fix the evaluation framework, gather the information and obtain an evaluation assessment by means of an evaluation process. The aim of any evaluation process is to obtain information about the worth of an item (product, service, material, etc.), and a complete description of different aspects, indicators, criteria in order to improve it or to compare it with other items with the purpose of identifying the best ones. The information gathered in evaluation processes is usually provided by a group of individuals, so called panel of experts, where each expert expresses his/her opinions about the evaluated items according to his/her own knowledge and perceptions.

Here, we focus on Sensory Evaluation 15,26,28,29, an evaluation discipline where the information provided by the panel of experts is perceived by the hu- man senses of sight, smell, taste, touch and hearing.

In evaluation processes the assessments provided by the panel of experts are usually expressed by numerical values when the evaluated criteria are quantitative in nature. However, in sensory evaluation the experts' knowledge is based on perceptions that are qualitative in nature and involve uncertainty and vagueness. In such cases, the use of linguistic assessments have provided successful results, in different areas such as "services evaluation and human resources management" 6,8, "marketing" 31 , "planning" 13 , "decision-making" 2,7,9,16,33, etc. The fuzzy linguistic approach ${ }^{32}$ provides a direct way to represent linguistic information by means of linguistic variables.

The feel that the panel of experts has more or less knowledge about the evaluated items is frequent. Therefore, the evaluation results could be improved if the experts were given a greater flexibility in the evaluation framework to express their preferences in different linguistic term sets. This paper proposes 
an evaluation model based on a decision analysis scheme to deal with sensory evaluation processes defined in a flexible framework composed by multigranular linguistic information.

The use of decision approaches have been successfully applied to solve evaluation problems in the literature $1,5,8,14,18,24,25$. In decision theory before making a decision it is carried out an analysis process that allows people to make decisions more consistently, i.e., it helps experts to deal with difficult decisions. The decision analysis is a suitable approach for evaluation processes because it helps to analyze the alternatives, criteria, indicators of the element/s under study that is the objective of the evaluation processes.

The University of Jaén is located in the southeast of Spain and it is the biggest olive oil producing area in the world. Recently the olive oil companies, in Jaén, have started to compete on international markets and they have realized how important it is to obtain and classify oil according to its quality in order to increase their profits and competitiveness. One way to control and improve the quality of oil is studying its properties by means of a sensory evaluation process. So far, in this process the experts have used precise numerical assessments, in spite of, they are expressing perceptions about the qualitative criteria obtained through their senses.

Therefore, the aim of this paper is to propose an evaluation model with multigranular linguistic frameworks to be applied to a sensory evaluation of olive oil, because the evaluated criteria about olive oil are better adapted to this type of framework.

In order to do that, this paper is structured as follows: Section 2 revises the scheme of the Decision Analysis and introduces a linguistic background revising in short the fuzzy linguistic approach, the 2tuple fuzzy linguistic representation model, and linguistic hierarchical contexts. Section 3 proposes a Sensory Evaluation Model that it is applied to the evaluation of olive oil in Section 4. Finally Section 5 points out some concluding remarks.

\section{Preliminaries}

In this section it is reviewed the scheme of the Decision Analysis used in our proposal of evaluation model, and it is made a short review of linguistic background that introduces necessary concepts to manage linguistic information and understand the proposed evaluation model.

\subsection{Decision Analysis}

The Decision Analysis is a discipline that pertains to Decision Theory, which helps the decision makers to reach a consistent decision in a decision making problem. The evaluation process can be modelled as different types of decision making problem. In this paper we model the evaluation process as a MultiExpert Decision Making (MEDM) problem, where decision makers express their opinions about a set alternatives by means of an utility vector. A classical decision analysis scheme consists of the following phases ${ }^{10}$ (see figure 1):

(i) Identify decision, objectives and alternatives of the problem.

(ii) Model: It defines the evaluation framework fixing the structure of the problem, in our case it is defined as a multigranular linguistic context in which the preferences will be assessed.

(iii) Gathering information: decision makers provide their information.

(iv) Rating alternatives: This phase obtains a collective value for each alternative.

(v) Choosing best alternatives: It selects the solution from the set of alternatives (applying a choice degree ${ }^{3,27}$ to the collective values computed in the previous phase).

(vi) Sensitive analysis: the solution obtained is analyzed in order to know if it is good enough to make a decision; otherwise, the solution goes back to the initial phases to improve the the quality of the results.

(vii) Make a decision.

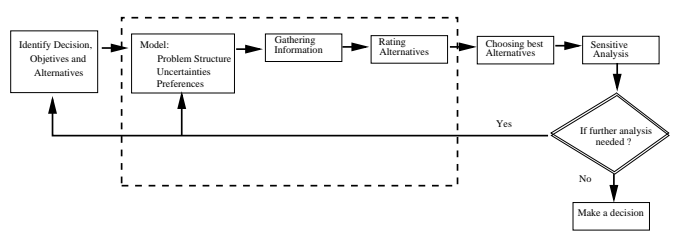

Fig. 1. Decision Analysis Scheme

The decision analysis involves more phases than those needed for an evaluation process. Therefore, the phases related to the evaluation problem that will be used in our proposal are those dashed in a rectangle in Fig. 1. 


\subsection{Linguistic Background}

Here we shall review some necessary concepts about linguistic information to understand our proposal. We review briefly the Fuzzy Linguistic Approach, the 2-tuple Linguistic representation model and the Linguistic Hierarchies.

\subsubsection{Fuzzy Linguistic Approach}

Many aspects of different activities in the real world cannot be assessed in a quantitative form, but rather in a qualitative one, i.e., with vague or imprecise knowledge. In that case, a better approach may be the use of linguistic assessments instead of numerical values. The fuzzy linguistic approach represents qualitative aspects as linguistic values by means of linguistic variables ${ }^{32}$.

We have to choose the appropriate linguistic descriptors for the term set and their semantics.

In the linguistic approach an important parameter to be determined is the "granularity of uncertainty" that indicates the degree of discrimination given by a term set, so that the more knowledge about the variable, the more granularity that can be utilized to assess it. When different experts have different uncertainty degrees about the items, then several linguistic term sets with a different granularity of uncertainty may be necessary.

One possibility of generating the linguistic term set consists in directly supplying the term set by considering all the terms distributed on a scale on which a total order ${ }^{30}$. For example, a set of seven terms $S$, could be:

$$
\left\{s_{0}: N, s_{1}: V L, s_{2}: L, s_{3}: M, s_{4}: H, s_{5}: V H, s_{6}: P\right\}
$$

Usually, in these cases, it is required that in the linguistic term set there exist:

(i) A negation operator: $\operatorname{Neg}\left(s_{i}\right)=s_{j}$ such that $j=g-\mathrm{i}$ ( $\mathrm{g}+1$ is the cardinality).

(ii) An order: $s_{i} \leq s_{j} \Longleftrightarrow i \leq j$. Therefore, there exists a min and a max operator.

The semantics of the terms are given by fuzzy numbers defined in the $[0,1]$ interval, which are usually described by membership functions. In this paper, we shall use as semantics of the linguistic terms triangular membership functions. whose representation is achieved by a 3-tuple $(a, b, c)$, where $b$ indicates the point in which the membership value is 1 , with $a$ and $c$ indicating the left and right limits of the definition domain of the membership function ${ }^{4}$. An example of uniformly linguistic term set may be:

$$
\begin{array}{lll}
P=(.83,1,1) & V H=(.67, .83,1) & H=(.5, .67, .83) \\
M=(.33, .5, .67) & L=(.17, .33, .5) & V L=(0, .17, .33) \\
N=(0,0, .17) . & &
\end{array}
$$

which is graphically shown in Figure 2.

We have observed that these fuzzy numbers adapt well to the sensory evaluation, but other fuzzy numbers could be used ${ }_{\mathrm{N}}^{21,22}$.

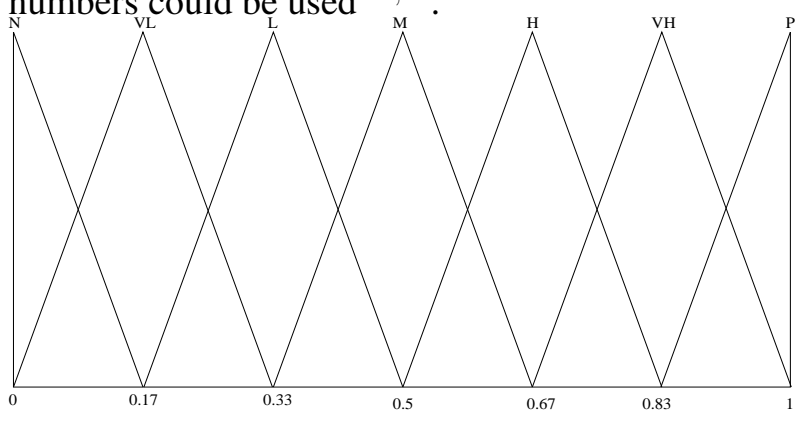

Fig. 2. A Set of 7 Terms with its Semantic

\subsubsection{2-tuple Linguistic Representation Model}

The use of linguistic variables always implies processes of Computing with Words $(\mathrm{CW})$. There exist different computational models to accomplish them. Classical CW models are those based on the Extension principle ${ }^{11}$ and symbolic one ${ }^{12}$. Both of them produce a loss of information in their computations 11,12 and hence a lack of precision in the results. Linguistic computational model ${ }^{19}$ based on linguistic 2-tuples carries out processes of $\mathrm{CW}$ in a precise way when the linguistic term sets are symmetrical and uniformly distributed.

The 2-tuple fuzzy linguistic representation model represents the linguistic information by means of a 2-tuple, $\left(s_{i}, \alpha\right)$, where $s$ is a linguistic label and $\alpha$ is a numerical value that represents the value of the symbolic translation.

Definition $1 .{ }^{20}$ Let $\beta$ be the result a symbolic aggregation operation in a linguistic term set, $S . \beta \in[0, g]$, being $g+1$ the cardinality of $S$. Let $i=\operatorname{round}(\beta)$ and $\alpha=\beta-i$ be two values, such that, $i \in[0, g]$ and $\alpha \in[-.5, .5)$ then $\alpha$ is called a Symbolic Translation.

This linguistic 2-tuple representation model defines a set of functions to make transformations between linguistic 2-tuples and numerical values: 
Definition 2. ${ }^{20}$ Let $S=\left\{s_{0}, \ldots, s_{g}\right\}$ be a linguistic term set and $\beta \in[0, g]$ a value supporting the result of a symbolic aggregation operation, then the 2-tuple that expresses the equivalent information to $\beta$ is obtained with the following function:

$$
\begin{gathered}
\Delta:[0, g] \longrightarrow S \times[-0.5,0.5) \\
\Delta(\beta)=\left\{\begin{array}{cc}
s_{i} & i=\operatorname{round}(\beta) \\
\alpha=\beta-i & \alpha \in[-.5, .5)
\end{array}\right.
\end{gathered}
$$

where round is the usual rounding operation, $s_{i}$ has the closest index label to " $\beta$ " and " $\alpha$ " is the value of the symbolic translation.

We note that $\Delta$ is bijective and $\Delta^{-1}: S \times$ $[-.5, .5) \longrightarrow[0, g]$ is defined as $\Delta^{-1}\left(s_{i}, \alpha\right)=i+\alpha$. In this way, the 2 -tuples of $S \times[-.5, .5)$ will be identified with the numerical values in the interval $[0, g]$.

Remark 1: From definitions 1 and 2, it is obvious that the conversion of a linguistic term into a linguistic 2 -tuple consists in adding a value 0 as symbolic translation:

$$
s_{i} \in S \Longrightarrow\left(s_{i}, 0\right)
$$

The 2-tuple representation model has associated a computational model presented in detail in [20].

\subsubsection{Linguistic Hierarchies}

As we have aforementioned we want to propose an evaluation model that deals with information defined in a multigranular linguistic framework. There exist different proposals to deal with this type of information ${ }^{17,21,23}$. In our proposal we shall use the hierarchical linguistic structure ${ }^{21}$ to improve the precision of the processes of $\mathrm{CW}$ in linguistic multigranular contexts.

\section{Building Linguistic Hierarchies}

A linguistic hierarchy is a set of levels, where each level is a linguistic term set with different granularity from the remaining of levels of the hierarchy. Each level belongs to a linguistic hierarchy is denoted as $\mathbf{l}(\mathbf{t}, \mathbf{n}(\mathbf{t}))$, being:

(i) $t$, indicates the level of the hierarchy,

(ii) $n(t)$, the granularity of the linguistic term set of the level $t$.
We assume levels containing linguistic terms whose membership functions are triangular-shaped, symmetrical and uniformly distributed in $[0,1]$. In addition, the linguistic term sets have an odd number of elements. The levels belonging to a linguistic hierarchy are ordered according to their granularity. For any two consecutive levels $t$ and $t+1$, $n(t+1)>n(t)$. This provides a linguistic refinement of the previous level.

From the above concepts, we define a linguistic hierarchy, $L H$, as the union of all levels $t: L H=$ $\bigcup_{t} l(t, n(t))$

Any set of term sets is not a $L H$. In [21] were defined rules in order to build proper $L H$.

Given a $L H, S^{n(t)}$ denotes the linguistic term set of $L H$ corresponding to the level $t$ of $L H$ with a granularity of uncertainty of $n(t): S^{n(t)}=$ $\left\{s_{0}^{n(t)}, \ldots, s_{n(t)-1}^{n(t)}\right\}$

The building of a linguistic hierarchy satisfies the following rules, which we call linguistic hierarchy basic rules:

(i) To preserve all former modal points of the membership functions of each linguistic term from one level to the following one.

(ii) To make smooth transitions between successive levels. The aim is to build a new linguistic term set, $S^{n(t+1)}$. A new linguistic term will be added between each pair of terms belonging to the term set of the previous level $t$. To carry out this insertion, we shall reduce the support of the linguistic labels in order to keep place for the new one located in the middle of them.

Generally, we can say that the linguistic term set of level $t+1 S^{n(t+1)}$ is obtained from its predecessor, $S^{n(t)}$, as: $l(t, n(t)) \rightarrow l(t+1,2 \cdot n(t)-1)$

A graphical example of a linguistic hierarchy is showed in Fig. 3: 


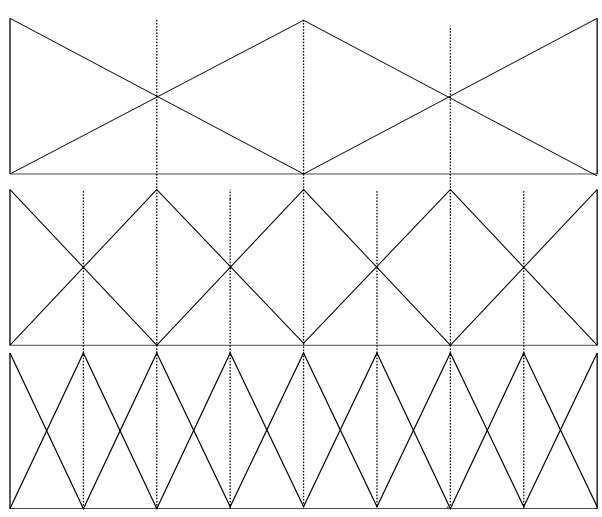

Fig. 3. Linguistic Hierarchy of 3,5 and 9 labels.

\section{CW Processes in LH}

Such as it was presented in [21] to accomplish $\mathrm{CW}$ processes in multigranular linguistic contexts. First, the information must be unified in an unique linguistic domain, so we will conduct these labels into an unique level of the LH, called basic representation level and noted as $t_{B R L}$ which will support the computational processes ${ }^{21}$. The labels provided by the experts, $\left(s_{i}^{n(t)}, \alpha\right) \in S^{n(t)} \times[-0.5,0.5)$, are transformed into linguistic 2-tuples in $S^{n\left(t_{B R L}\right)}$ by means of the transformation functions, $T F_{t_{B R L}}^{t}$.

$$
\begin{gathered}
T F_{t^{\prime}}^{t}: l(t, n(t)) \longrightarrow l\left(t^{\prime}, n\left(t^{\prime}\right)\right) \\
T F_{t^{\prime}}^{t}\left(s_{i}^{n(t)}, \alpha^{n(t)}\right)=\Delta\left(\frac{\Delta^{-1}\left(s_{i}^{n(t)}, \alpha^{n(t)}\right) \cdot\left(n\left(t^{\prime}\right)-1\right)}{n(t)-1}\right)
\end{gathered}
$$

The reason to fix the $L H$ basic rules for their building is that those rules guarantee that the $\mathrm{CW}$ processes in $L H$ can be carried out without loss of information. To do so, it is necessary the use of the 2-tuple computacional model ${ }^{20}$ and the use of a transformation function defined in [21].

Proof $\mathbf{1}^{21}$. The transformation function, $T F_{t^{\prime}}^{t}$, between linguistic terms in different levels of the linguistic hierarchy, $L H$, is bijective:

$$
T F_{t}^{t^{\prime}}\left(T F_{t^{\prime}}^{t}\left(s_{i}^{n(t)}, \alpha^{n(t)}\right)\right)=\left(s_{i}^{n(t)}, \alpha^{n(t)}\right)
$$

\section{Sensory Evaluation Model dealing with Multi- granular Linguistic Information}

Our aim is to propose a Sensory Evaluation model dealing with different linguistic scales and based on the linguistic decision analysis ${ }^{10}$, whose mathematical formalism will be the linguistic 2-tuple model in order to obtain accurate and reliable evaluation results in multigranular linguistic evaluation frameworks; so that it can be applied to the olive oil sensory evaluation process.

The decision analysis scheme that will use our proposal for the sensory evaluation model consists of the following phases (graphically, Fig. 4) revised in section:

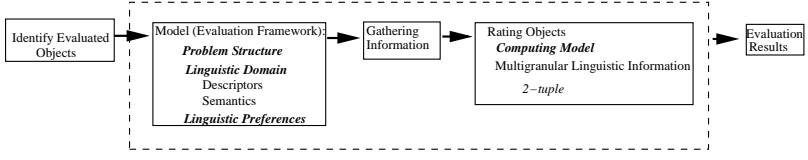

Fig. 4. Sensory Evaluation Scheme based on linguistic 2-tuple decision analysis

(i) Identify Evaluated Objects.

(ii) Evaluation Framework.

(iii) Gathering Information.

(iv) Rating Objects.

(v) Evaluation Results.

The following subsections present in detail the main phases of the above linguistic sensory evaluation model.

\subsection{Evaluation Framework}

This phase defines the evaluation framework, such that, the problem structure is defined and the linguistic descriptors and semantics that will be used by the experts to express their information about the sensory features of the evaluated objects are chosen.

First of all, it analyzes the features that will be evaluated. The selection of the linguistic term sets utilized to assess those features will depend on the knowledge and experience of each expert of the panel. Therefore, the granularity of the linguistic term sets are chosen according to the experts' knowledge. We propose a multigranular linguistic evaluation framework based on a MEDM problem structure where the experts can express their opinions by means of linguistic labels belonging to the term sets of a $L H$. Hence the evaluation framework will be as the following one:

$E=\left\{e_{1}, \ldots, e_{n}\right\}$, a panel of experts.

$X=\left\{x_{1}, \ldots, x_{m}\right\}$ set of items to be evaluated sensorially.

$S^{n(t)}$, linguistic term sets in $L H$. 
$F=\left\{f_{1}, \ldots, f_{h}\right\}$ set of sensory features that characterizes each evaluated item $x_{i}$

\subsection{Gathering Information}

Once the framework has been defined in order to evaluate the different items. The evaluation process must obtain the knowledge from the panel of experts.

Due to the fact that, the framework fixed above is based on the MEDM ${ }^{21}$ problem structure where the experts will provide their knowledge on utility vectors that contain a linguistic assessment for each evaluated feature. Each expert, $e_{i}$ provides his/her preferences in $S_{i}^{n(t)}$ by means of an utility vector:

$$
U_{i}=\left\{u_{11}^{i}, \ldots, u_{1 h}^{i}, u_{21}^{i}, \ldots, u_{2 h}^{i}, \ldots, u_{m 1}^{i}, \ldots, u_{m h}^{i}\right\}
$$

where $u_{j k}^{i} \in S_{i}^{n(t)}$ is the assessment provided to the feature $f_{k}$ of the item $x_{j}$ by the expert $e_{i}$. Consequently, in the gathering process every $e_{i}$ will provide his/her utility vector $U_{i}$ expressed by linguistic labels in a linguistic term set in the $L H$.

\subsection{Rating Objects}

The aim of the sensory evaluation process is to obtain information about the worth of an evaluated item, so this phase of the evaluation model computes a global value for each item according to the information gathered in the previous phase.

To compute such a global value, first the information gathered is expressed by means of linguistic 2-tuples using the Remark 1.

$$
u_{j k}^{i} \Rightarrow\left(u_{j k}^{i}, 0\right)
$$

Given that we cannot compute directly with the multigranular linguistic information provided by the panel of experts, it must be conducted into one expression domain, $S^{n\left(t_{B R L}\right)}$ by means of the transformation functions, $T F_{t_{B R L}}^{t}$. Once the information has been conducted, our model will apply a two-step aggregation process to compute a global evaluation for the evaluated item:

(i) Computing collective evaluations for each feature: first, the rating process will compute a collective linguistic 2-tuple, $\left(u_{j k}, \alpha\right)$, for each feature, $f_{k}$, of the object $x_{j}$, using an aggregation operator, $A G O P_{1}$, on the assessments, $u_{j k}^{i}$, provided by all the experts, $e_{i}$, and represented in $t_{B R L}$ :

$$
\begin{gathered}
\left(u_{j k}, \alpha\right)=A G O P_{1}\left(\left(u_{j k}^{1}, \alpha_{1}\right), \ldots,\left(u_{j k}^{n}, \alpha_{n}\right)\right) \\
u_{j k} \in S^{n\left(t_{B R L}\right)}
\end{gathered}
$$

(ii) Computing a collective evaluation for each object: the final aim of the rating process is to obtain a global evaluation, $\left(u_{j}, \alpha\right)$, for each evaluated object, $x_{j}$ according to all the experts and features that take part in the sensory evaluation process. To do so, this process will aggregate the collective linguistic 2-tuples for each feature, $\left(u_{j k}, \alpha\right)$, using an aggregation operator, $\mathrm{AGOP}_{2}$ :

$$
\begin{gathered}
\left(u_{j}, \alpha\right)=A G O P_{2}\left(\left(u_{j 1}, \alpha_{1}\right), \ldots .,\left(u_{j h}, \alpha_{h}\right)\right) \\
u_{j} \in S^{t_{B R L}}
\end{gathered}
$$

The aggregation operators, $A G O P_{1}$ and $A G O P_{2}$, could be the same or different ones depending on each sensory evaluation problem.

The aggregation results will be expressed in $S^{n\left(t_{B R L}\right)}$, in our model it is not necessary to express them in the initial expression domain, $S^{n(t)}$. If the experts required it, in such a model it shall be applied the transformation function $T F_{t}^{t_{B R L}}$ to the results, $\left(u_{j}, \alpha\right)$ obtained by $\mathrm{AGOP}_{2}$ in order to return the results to each user in his/her initial expression domain.

\section{Olive Oil Sensory Evaluation}

The virgin olive oil is distinguished for the rest of vegetal oils, because of its special organoleptical properties of color, scent and flavor. The evaluation of their organoleptical properties of scent and flavor are determined by means of the application of the sensorial analysis. The sensorial evaluation is used as a measure of quality of the oil and identifies its different categories (extra virgin, virgin, ordinary virgin or lampante) *

Currently, the classification of olive oil samples is made according to the intensity of the defects and positive attributes (see Table 1) given by a group of

*URL:http://www.internationaloliveoil.org/downloads/orga6.pdf 
experts selected and trained as a panel. The experts fill in the profile sheet showed in Fig. 5, with quantitative values according to the intensity of their perceptions about each of the negative and positive attributes.

Table 1. Attributes that the panel of experts can evaluate in Olive oil.

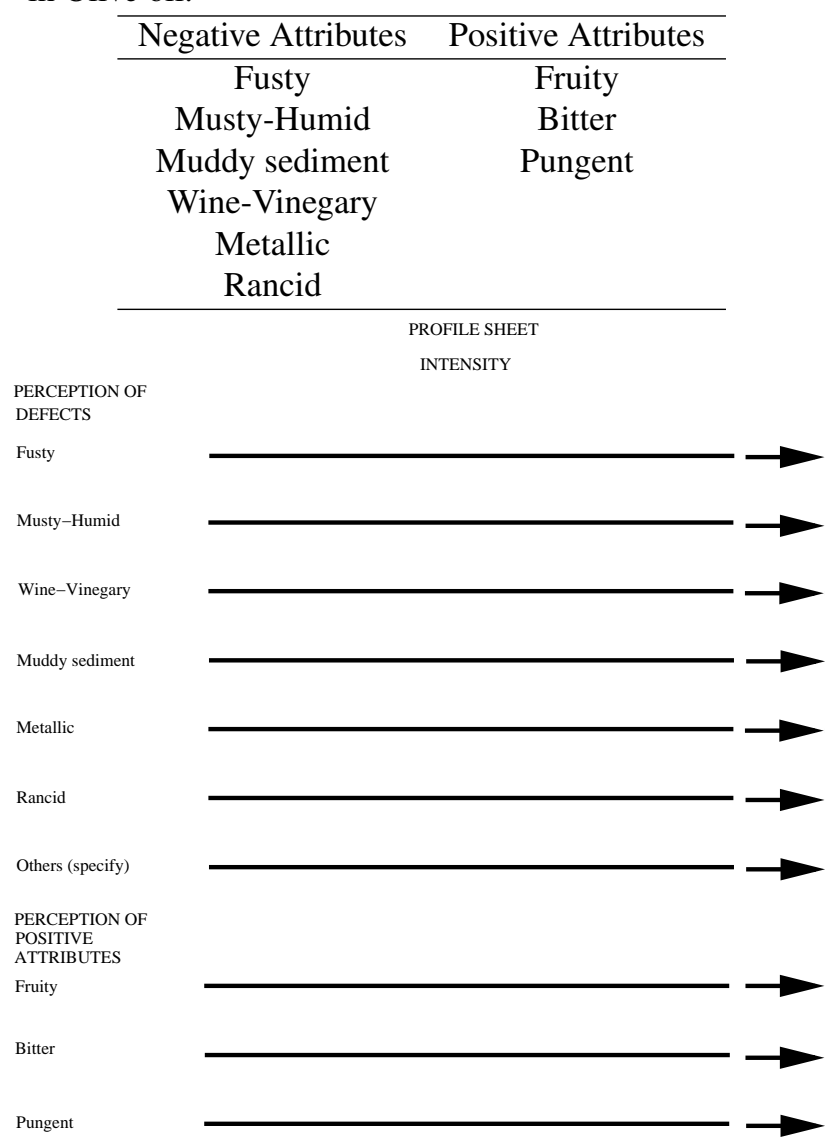

Fig. 5. Profile sheet

According to the intensity of the defects and positive attributes of the Olive oil, this shall be classified:

- The extra virgin grade when the median of the defects is equal to 0 and the median of the fruity attribute is more than 0 .

- The virgin grade when the median of the defects is more than 0 and less than or equal to 2.5 and the median of the fruity attribute is more than 0 .

- The ordinary virgin grade when the median of the defects is more than 2.5 and less than or equal to 6.0 or when the median of the defects is less than or equal to 2.5 and the median of the fruity attribute is equal to 0 .

- The lampante virgin grade when the median of the defects is more than 6.0.

Our aim is to define a proper qualitative framework for the olive oil sensory evaluation. Due to the fact that, the experts of panel can have more or less knowledge degree depending on the evaluated properties, we shall propose a multigranular linguistic framework for this evaluation and apply the model proposed in section 3 to classify olive oil samples in such an evaluation framework. To do so, we present the specific evaluation phases for our proposal.

\subsection{Evaluation Framework}

First, we have to define the framework based on multigranular information to evaluate olive oil samples. After a survey with different connoisseurs, the scales chosen to assess in a qualitative way the intensity of attributes os Table 1 showed in Fig. 6 and 7 that are part of the $L H, l(3,9)$, showed in Fig. 3.



Fig. 6. Scale 1 for the sensory evaluation in $\mathrm{LH}$

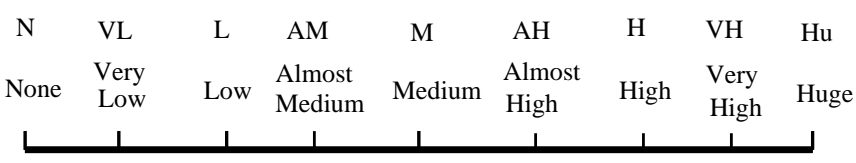

Fig. 7. Scale 2 for the sensory evaluation in LH

In order to understand both easily the framework and the remaining phases of the model, we present and solve an example. Let's suppose an Olive Oil Tasting Panel of eight connoisseurs $E=$ $\left\{e_{1}, \ldots, e_{8}\right\}$ will evaluate the intensity of the negative attributes: Fusty, Musty-Humid, Muddy sediment, Wine-Vinegary, Metallic, Rancid and positive attributes Fruity, Bitter, Pungent. That will be noted as $F=\left\{f_{1}, \ldots, f_{9}\right\}$ set of sensorial attributes of a sample of Olive Oil. According to the evaluation model proposed the evaluation process carries out the following phases.

\subsection{Gathering Information}

In our qualitative framework the Tasting Panel provides their knowledge about the attributes of Table 
1 using the profile sheet in Fig.8. The preferences provided by the experts are showed in Table 2 :

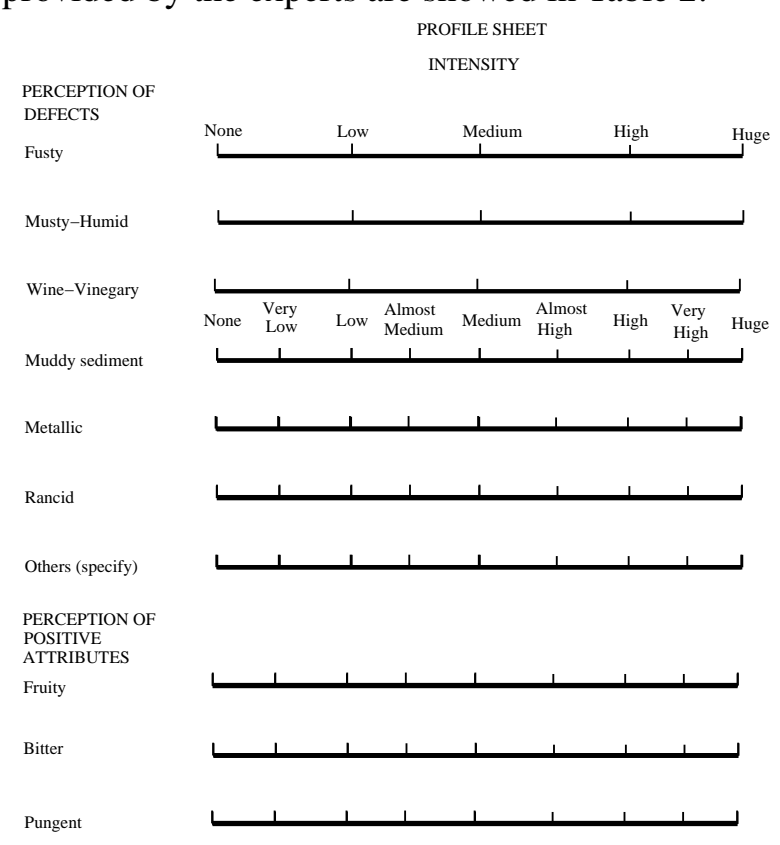

Fig. 8. Profile sheet

In order to accomplish $\mathrm{CW}$ processes the experts' preferences will be transformed into 2-tuple representation model to manage easily this information, the results of this transformation is showed in Table 3.

The experts' preferences are transformed into linguistic 2-tuples in $l(3,9)$ by means of the transformation function, $T F_{3}^{2}$, the results of this transformation is showed in Table 4.

\subsection{Rating Objects}

The classification of olive oil samples is carried out according to the intensity of the defects and the fruity attributes. To do so, the proposed model computes a collective evaluation of the evaluated features; in our case, these are the negative features and the fruity feature.

In the quantitative model this collective value is computed using the median operator, so in our proposal we shall extend the median operator to deal with linguistic 2-tuples that is defined as follows:

Definition 3. Let $X=\left\{\left(s_{j}, \alpha\right)_{1}, \ldots,\left(s_{j}, \alpha\right)_{n}\right\}, s_{j} \in$ $S=\left\{s_{0}, \ldots, s_{g}\right\}$ be an ordered set of 2-tuples and $\left(s_{j}, \alpha\right)_{k}$ is the $k$-th largest of the elements in $X$, the 2-tuple median operator Med $(X)$ is computed as,

$$
\operatorname{Med}(X)=\left\{\begin{array}{c}
\operatorname{Med}(X)=\left(s_{j}, \alpha\right)_{\frac{n+1}{2}} \text { if } n \text { is odd } \\
\operatorname{Med}(X)=\left(s_{j}, \alpha\right)_{\frac{n}{2}} \text { if } n \text { is even }
\end{array}\right.
$$

When $n$ is even the value of the median is not unique, $\operatorname{Med}(X) \in\left[\left(s_{j}, \alpha\right)_{\frac{n}{2}},\left(s_{j}, \alpha\right)_{\frac{n+1}{2}}\right]$. More generally:

$$
\operatorname{Med}(X)=\Delta\left(\frac{\Delta^{-1}\left(s_{j}, \alpha\right)_{\frac{n}{2}}+\Delta^{-1}\left(s_{j}, \alpha\right)_{\frac{n+1}{2}}}{2}\right)
$$

The rating process consists of two aggregation steps:

1. Computing collective evaluations for each feature: We can use the aggregation operator for 2-tuples $\Lambda^{F}$, being $F=\operatorname{Med}(X)$ i.e., the median for 2-tuples defined above. In Table 5 we see the collective values obtained by the median for each feature.

An example of the computation of the median for the positive fruity attribute, $f_{7}$, is:

$$
\begin{gathered}
\Lambda^{F}\left[\left(s_{1}^{9}, 0\right),\left(s_{1}^{9}, 0\right),\left(s_{2}^{9}, 0\right),\left(s_{2}^{9}, 0\right),\left(s_{2}^{9}, 0\right)\right. \\
\left.\left(s_{2}^{9}, 0\right),\left(s_{1}^{9}, 0\right),\left(s_{1}^{9}, 0\right)\right]=\left(s_{2}^{9},-.5\right)
\end{gathered}
$$

2. Computing a collective evaluation for each object: in the olive oil sensory evaluation this process will obtain two different values one for the negative features, noted as $d$, and another one noted as $p$ for the fruity attribute. Those values will be computed as: $d=$ $\max \left(g_{i}, \ldots, g_{j}\right)$, with $g_{i}=\Lambda^{F}\left(f_{i}\right)$ the median for the negative features $f_{i}, i \in\{1,2,3,4,5,6\}$ and $p=\Lambda^{F}\left(f_{7}\right)$, the median for the fruity feature. From these values the olive oil sample will be classified according to the classifier, $\operatorname{cl}(x, y)$, which used the values $d$ and $p$ as $c l(d, p)$ (see Fig. 9)

In our example:

$d=\max \left\{\left(s_{0}^{9}, 0\right),\left(s_{1}^{9}, 0\right),\left(s_{0}^{9}, 0\right),\left(s_{0}^{9}, 0\right),\left(s_{0}^{9}, 0\right),\left(s_{1}^{9},-.5\right)\right\}=$

$$
\left(s_{1}^{9}, 0\right),
$$

and $p=\left(s_{2}^{9},-.5\right)$ then

$$
\operatorname{cl}\left(\left(s_{1}^{9}, 0\right),\left(s_{2}^{9},-.5\right)\right) \Rightarrow \text { virgin. }
$$

Then, the classification for the sample of olive oil is virgin.

\section{Conclusions}


Table 2: Olive Oil Tasting Panel's utility vectors for the attributes.

\begin{tabular}{c|ccccccccc}
\hline & $f_{1}$ & $f_{2}$ & $f_{3}$ & $f_{4}$ & $f_{5}$ & $f_{6}$ & $f_{7}$ & $f_{8}$ & $f_{9}$ \\
\hline$e_{1}$ & $N^{\prime}$ & $N^{\prime}$ & $N^{\prime}$ & $N$ & $N$ & $N$ & $V L$ & $N$ & $N$ \\
$e_{2}$ & $N^{\prime}$ & $N^{\prime}$ & $N^{\prime}$ & $N$ & $N$ & $V L$ & $V L$ & $N$ & $N$ \\
$e_{3}$ & $N^{\prime}$ & $L^{\prime}$ & $N^{\prime}$ & $N$ & $V L$ & $L$ & $L$ & $N$ & $N$ \\
$e_{4}$ & $N^{\prime}$ & $L^{\prime}$ & $N^{\prime}$ & $N$ & $N$ & $V L$ & $L$ & $N$ & $N$ \\
$e_{5}$ & $N^{\prime}$ & $L^{\prime}$ & $N^{\prime}$ & $N$ & $N$ & $N$ & $L$ & $N$ & $N$ \\
$e_{6}$ & $N^{\prime}$ & $L^{\prime}$ & $N^{\prime}$ & $N$ & $N$ & $V L$ & $L$ & $N$ & $N$ \\
$e_{7}$ & $N^{\prime}$ & $N^{\prime}$ & $N^{\prime}$ & $N$ & $N$ & $N$ & $V L$ & $N$ & $N$ \\
$e_{8}$ & $N^{\prime}$ & $N^{\prime}$ & $N^{\prime}$ & $N$ & $N$ & $M$ & $V L$ & $N$ & $N$ \\
\hline
\end{tabular}

Table 3: Olive Oil Tasting Panel's utility vectors for the feature the 2-tuple representation model.

\begin{tabular}{c|ccccccccc}
\hline & $f_{1}$ & $f_{2}$ & $f_{3}$ & $f_{4}$ & $f_{5}$ & $f_{6}$ & $f_{7}$ & $f_{8}$ & $f_{9}$ \\
\hline$e_{1}$ & $\left(s_{0}^{5}, 0\right)$ & $\left(s_{0}^{5}, 0\right)$ & $\left(s_{0}^{5}, 0\right)$ & $\left(s_{0}^{9}, 0\right)$ & $\left(s_{0}^{9}, 0\right)$ & $\left(s_{0}^{9}, 0\right)$ & $\left(s_{1}^{9}, 0\right)$ & $\left(s_{0}^{9}, 0\right)$ & $\left(s_{0}^{9}, 0\right)$ \\
$e_{2}$ & $\left(s_{0}^{5}, 0\right)$ & $\left(s_{0}^{5}, 0\right)$ & $\left(s_{0}^{5}, 0\right)$ & $\left(s_{0}^{9}, 0\right)$ & $\left(s_{0}^{9}, 0\right)$ & $\left(s_{1}^{9}, 0\right)$ & $\left(s_{1}^{9}, 0\right)$ & $\left(s_{0}^{9}, 0\right)$ & $\left(s_{0}^{9}, 0\right)$ \\
$e_{3}$ & $\left(s_{0}^{5}, 0\right)$ & $\left(s_{1}^{5}, 0\right)$ & $\left(s_{0}^{5}, 0\right)$ & $\left(s_{0}^{9}, 0\right)$ & $\left(s_{1}^{9}, 0\right)$ & $\left(s_{2}^{9}, 0\right)$ & $\left(s_{2}^{9}, 0\right)$ & $\left(s_{0}^{9}, 0\right)$ & $\left(s_{0}^{9}, 0\right)$ \\
$e_{4}$ & $\left(s_{0}^{5}, 0\right)$ & $\left(s_{1}^{5}, 0\right)$ & $\left(s_{0}^{5}, 0\right)$ & $\left(s_{0}^{9}, 0\right)$ & $\left(s_{0}^{9}, 0\right)$ & $\left(s_{1}^{9}, 0\right)$ & $\left(s_{2}^{9}, 0\right)$ & $\left(s_{0}^{9}, 0\right)$ & $\left(s_{0}^{9}, 0\right)$ \\
$e_{5}$ & $\left(s_{0}^{5}, 0\right)$ & $\left(s_{1}^{5}, 0\right)$ & $\left(s_{0}^{5}, 0\right)$ & $\left(s_{0}^{9}, 0\right)$ & $\left(s_{0}^{9}, 0\right)$ & $\left(s_{0}^{9}, 0\right)$ & $\left(s_{2}^{9}, 0\right)$ & $\left(s_{0}^{9}, 0\right)$ & $\left(s_{0}^{9}, 0\right)$ \\
$e_{6}$ & $\left(s_{0}^{5}, 0\right)$ & $\left(s_{1}^{5}, 0\right)$ & $\left(s_{0}^{5}, 0\right)$ & $\left(s_{1}^{9}, 0\right)$ & $\left(s_{0}^{9}, 0\right)$ & $\left(s_{1}^{9}, 0\right)$ & $\left(s_{2}^{9}, 0\right)$ & $\left(s_{0}^{9}, 0\right)$ & $\left(s_{0}^{9}, 0\right)$ \\
$e_{7}$ & $\left(s_{0}^{5}, 0\right)$ & $\left(s_{0}^{5}, 0\right)$ & $\left(s_{0}^{5}, 0\right)$ & $\left(s_{0}^{9}, 0\right)$ & $\left(s_{0}^{9}, 0\right)$ & $\left(s_{0}^{9}, 0\right)$ & $\left(s_{1}^{9}, 0\right)$ & $\left(s_{0}^{9}, 0\right)$ & $\left(s_{0}^{9}, 0\right)$ \\
$e_{8}$ & $\left(s_{0}^{5}, 0\right)$ & $\left(s_{0}^{5}, 0\right)$ & $\left(s_{0}^{5}, 0\right)$ & $\left(s_{0}^{9}, 0\right)$ & $\left(s_{0}^{9}, 0\right)$ & $\left(s_{0}^{9}, 0\right)$ & $\left(s_{1}^{9}, 0\right)$ & $\left(s_{0}^{9}, 0\right)$ & $\left(s_{0}^{9}, 0\right)$ \\
\hline
\end{tabular}

Table 4: Olive Oil Tasting Panel's utility vectors in linguistic 2-tuples in $S^{9}$

\begin{tabular}{c|ccccccccc}
\hline & $f_{1}$ & $f_{2}$ & $f_{3}$ & $f_{4}$ & $f_{5}$ & $f_{6}$ & $f_{7}$ & $f_{8}$ & $f_{9}$ \\
\hline$e_{1}$ & $\left(s_{0}^{9}, 0\right)$ & $\left(s_{0}^{9}, 0\right)$ & $\left(s_{0}^{9}, 0\right)$ & $\left(s_{0}^{9}, 0\right)$ & $\left(s_{0}^{9}, 0\right)$ & $\left(s_{0}^{9}, 0\right)$ & $\left(s_{1}^{9}, 0\right)$ & $\left(s_{0}^{9}, 0\right)$ & $\left(s_{0}^{9}, 0\right)$ \\
$e_{2}$ & $\left(s_{0}^{9}, 0\right)$ & $\left(s_{0}^{9}, 0\right)$ & $\left(s_{0}^{9}, 0\right)$ & $\left(s_{0}^{9}, 0\right)$ & $\left(s_{0}^{9}, 0\right)$ & $\left(s_{1}^{9}, 0\right)$ & $\left(s_{1}^{9}, 0\right)$ & $\left(s_{0}^{9}, 0\right)$ & $\left(s_{0}^{9}, 0\right)$ \\
$e_{3}$ & $\left(s_{0}^{9}, 0\right)$ & $\left(s_{2}^{9}, 0\right)$ & $\left(s_{0}^{9}, 0\right)$ & $\left(s_{0}^{9}, 0\right)$ & $\left(s_{1}^{9}, 0\right)$ & $\left(s_{2}^{9}, 0\right)$ & $\left(s_{2}^{9}, 0\right)$ & $\left(s_{0}^{9}, 0\right)$ & $\left(s_{0}^{9}, 0\right)$ \\
$e_{4}$ & $\left(s_{0}^{9}, 0\right)$ & $\left(s_{2}^{9}, 0\right)$ & $\left(s_{0}^{9}, 0\right)$ & $\left(s_{0}^{9}, 0\right)$ & $\left(s_{0}^{9}, 0\right)$ & $\left(s_{1}^{9}, 0\right)$ & $\left(s_{2}^{9}, 0\right)$ & $\left(s_{0}^{9}, 0\right)$ & $\left(s_{0}^{9}, 0\right)$ \\
$e_{9}$ & $\left(s_{0}^{9}, 0\right)$ & $\left(s_{2}^{9}, 0\right)$ & $\left(s_{0}^{9}, 0\right)$ & $\left(s_{0}^{9}, 0\right)$ & $\left(s_{0}^{9}, 0\right)$ & $\left(s_{0}^{9}, 0\right)$ & $\left(s_{2}^{9}, 0\right)$ & $\left(s_{0}^{9}, 0\right)$ & $\left(s_{0}^{9}, 0\right)$ \\
$e_{6}$ & $\left(s_{0}^{9}, 0\right)$ & $\left(s_{2}^{9}, 0\right)$ & $\left(s_{0}^{9}, 0\right)$ & $\left(s_{1}^{9}, 0\right)$ & $\left(s_{0}^{9}, 0\right)$ & $\left(s_{1}^{9}, 0\right)$ & $\left(s_{2}^{9}, 0\right)$ & $\left(s_{0}^{9}, 0\right)$ & $\left(s_{0}^{9}, 0\right)$ \\
$e_{7}$ & $\left(s_{0}^{9}, 0\right)$ & $\left(s_{0}^{9}, 0\right)$ & $\left(s_{0}^{9}, 0\right)$ & $\left(s_{0}^{9}, 0\right)$ & $\left(s_{0}^{9}, 0\right)$ & $\left(s_{0}^{9}, 0\right)$ & $\left(s_{1}^{9}, 0\right)$ & $\left(s_{0}^{9}, 0\right)$ & $\left(s_{0}^{9}, 0\right)$ \\
$e_{8}$ & $\left(s_{0}^{9}, 0\right)$ & $\left(s_{0}^{9}, 0\right)$ & $\left(s_{0}^{9}, 0\right)$ & $\left(s_{0}^{9}, 0\right)$ & $\left(s_{0}^{9}, 0\right)$ & $\left(s_{0}^{9}, 0\right)$ & $\left(s_{1}^{9}, 0\right)$ & $\left(s_{0}^{9}, 0\right)$ & $\left(s_{0}^{9}, 0\right)$ \\
\hline
\end{tabular}

Table 5: Olive Oil Tasting Panel's utility vectors in linguistic 2-tuples in $S^{9}$

\begin{tabular}{c|ccccccccc}
\hline & $f_{1}$ & $f_{2}$ & $f_{3}$ & $f_{4}$ & $f_{5}$ & $f_{6}$ & $f_{7}$ & $f_{8}$ & $f_{9}$ \\
\hline$\Lambda^{F}\left(f_{i}\right)$ & $\left(s_{0}^{9}, 0\right)$ & $\left(s_{1}^{9}, 0\right)$ & $\left(s_{0}^{9}, 0\right)$ & $\left(s_{0}^{9}, 0\right)$ & $\left(s_{0}^{9}, 0\right)$ & $\left(s_{1}^{9},-.5\right)$ & $\left(s_{2}^{9},-.5\right)$ & $\left(s_{0}^{9}, 0\right)$ & $\left(s_{0}^{9}, 0\right)$ \\
\hline
\end{tabular}

Figure 9: Function cl(d,p)

$$
c l(d, p)=\left\{\begin{array}{cc}
\text { extra virgin } & d=\left(s_{0}^{9}, 0\right) \text { and } p>\left(s_{0}^{9}, 0\right) \\
\text { virgin } & \left(s_{0}^{9}, 0\right)<d \leq\left(s_{2}^{9}, 0\right) \text { and } p>\left(s_{0}, 0\right) \\
\text { ordinaryvirgin } & \left(s_{2}^{9}, 0\right)<d \leq\left(s_{5}^{9}, 0\right) \text { ord } \leq\left(s_{2}^{9}, 0\right) \text { and } p=\left(s_{0}^{9}, 0\right) \\
\text { lampante virgin } & d>\left(s_{5}^{9}, 0\right)
\end{array}\right.
$$


The sensory evaluation is a process in which the information provided by the panel of experts involves uncertainty because it is acquired via human senses. Usually, this information is vague and uncertain, so a qualitative modelling is quite suitable. Additionally, the panel of experts can have more or less knowledge degree depending on the evaluated items. In this paper, we have presented a sensory evaluation model that offers a multigranular linguistic evaluation framework to the experts in order to offer a greater flexibility to express their knowledge and obtain better results in the evaluation process. And it has been applied to a simple example of olive oil sensory evaluation model in order to show its utility.

\section{Acknowledgments}

This paper has been partially supported by the research project TIN2006-02121 and Feder Fonds.

\section{References}

1. J. Antes, L. Campen, U. Derigs, C Titze, and G.D. Wolle. A model-based decision support system for the evaluation of flight schedules for cargo airlines. Decision Support Systems, 22(4):307-323, 1998.

2. B. Arfi. Fuzzy decision making in politics: A linguistic fuzzy-set approach (lfsa). Political Analysis, 13(1):23-56, 2005.

3. K.J. Arrow. Social Choice and Individual Values. Yale University Press, New Haven CT, 1963.

4. P.P. Bonissone and K.S. Decker. Selecting Uncertainty Calculi and Granularity: An Experiment in TradingOff Precision and Complexity. In L.H. Kanal and J.F. Lemmer, Editors., Uncertainty in Artificial Intelligence. North-Holland, 1986.

5. D. Bouyssou, T. Marchant, M. Pirlot, P. Perny, and A. Tsoukia's. Evaluation and Decision Models: A critical perspective. Kluwer Academic Publishers, 2000.

6. Y-L. Kuo C-H. Yeh. Evaluating passenger services of asia-pacific international airports. Transportation Research Part E: Logistic and Transportantion Review, 39(1):35-48, 2003.

7. S.L. Chang, R.C. Wang, and S.Y. Wang. Applying fuzzy linguistic quantifier to select supply chain partners at different phases of product life cycle. International Journal of Production Economics, 100(2):348359, 2006.

8. C.T. Chen. Applying linguistic decision-making method to deal with service quality evaluation problems. International Journal of Uncertainty, Fuzziness and Knowledge-Based Systems, 9(Suppl.):103$114,2001$.
9. C.H. Cheng and Y. Lin. Evaluating the best main battle tank using fuzzy decision theory with linguistic criteria evaluation. European Journal of Operational Research, 142:174-186, 2002.

10. R.T. Clemen. Making Hard Decisions. An Introduction to Decision Analisys. Duxbury Press, 1995.

11. R. Degani and G. Bortolan. The problem of linguistic approximation in clinical decision making. International Journal of Approximate Reasoning, 2:143-162, 1988.

12. M. Delgado, J.L. Verdegay, and M.A Vila. On aggregation operations of linguistic labels. International Journal of Intelligent Systems, 8:351-370, 1993.

13. G. Devedzic. Linguistic and choquet integral based evaluation in process planning. International Journal of Uncertainty, Fuzziness and Knowledge-Based Systems, 9(Suppl.):115-127, 2001.

14. G.B. Devedzic and E. Pap. Multicriteria-multistages linguistic evaluation and ranking of machine tools. Fuzzy Sets and Systems, 102:451-461, 1999.

15. G.B. Dijksterhuis. Multivariate Data Analysis in Sensory and Consumer Science, Food and Nutrition. Press Inc. Trumbull, Connecticut, USA, 1997.

16. F. Herrera and E. Herrera-Viedma. Linguistic decision analysis: Steps for solving decision problems under linguistic information. Fuzzy Sets and Systems, 115:67-82, 2000.

17. F. Herrera, E. Herrera-Viedma, and L. Martínez. A fusion approach for managing multi-granularity linguistic terms sets in decision making. Fuzzy Sets and Systems, 114(1):43-58, 2000.

18. F. Herrera, E. Herrera-Viedma, L. Martinez, F. Mata, and P.J. Sanchez. A Multi-Granular Linguistic Decision Model for Evaluating the Quality of Network Services. Intelligent Sensory Evaluation: Methodologies and Applications. Springer, Ruan Da, Zeng Xianyi (Eds.), 2004.

19. F. Herrera and L. Martínez. A selection method based on the 2-tuple linguistic representation model for decision-making with multi-granularity linguistic information. In Proceedings of the EUSFLATESTYLF Joint Conference 99, pages 453-456, Palma de Mallorca (Spain), September 1999.

20. F. Herrera and L. Martínez. A 2-tuple fuzzy linguistic representation model for computing with words. IEEE Transactions on Fuzzy Systems, 8(6):746-752, 2000.

21. F. Herrera and L. Martínez. A model based on linguistic 2-tuples for dealing with multigranularity hierarchical linguistic contexts in multiexpert decisionmaking. IEEE Transactions on Systems, Man and Cybernetics. Part B: Cybernetics, 31(2):227-234, 2001.

22. F. Herrera, L. Martínez, and P.J. Sánchez. Managing non-homogeneous information in group decision making. European Journal of Operational Research, 2004.

23. E. Herrera-Viedma, L. Martínez, F. Mata, and F Chiclana. A consensus support system model for group 
decision-making problems with multi-granular linguistic preference relations. IEEE Transactions on Fuzzy Systems, 13(5):644-658, 2005.

24. A. Jiménez, S. Ríos-Insua, and A. Mateos. A decision support system for multiattribute utility evaluation based on imprecise assignments. Decision Support Systems, 36(1):65-79, 2003.

25. A.C. Marquez and C. Blanchar. A decision support system for evaluating operations investments in high-technology business. Decision Support Systems, 41(2):472-487, 2006.

26. L. Martínez. Sensory evaluation based on linguistic decision analysis. International Journal of Aproximated Reasoning, 44 Num 2:148-164, 2007.

27. S.A. Orlovsky. Decision-making with a fuzzy preference relation. Fuzzy Sets Systems, 1:155-167, 1978.

28. D. Ruan and X. Zeng (Eds.). Sensory Evaluation: Methodologies and Applications. Springer, 2004.
29. H. Stone and J.L. Sidel. Sensory Evaluation Practice. Academic Press Inc., San Diego, CA, 1993.

30. R.R. Yager. An approach to ordinal decision making. International Journal of Approximate Reasoning, 12:237-261, 1995.

31. R.R. Yager, L.S. Goldstein, and E. Mendels. Fuzmar: An approach to aggregating market research data based on fuzzy reasoning. Fuzzy Sets and Systems, 68:1-11, 1994.

32. L.A. Zadeh. The concept of a linguistic variable and its applications to approximate reasoning. Information Sciences, Part I, II, III, 8,8,9:199-249,301-357,4380, 1975.

33. Xu Z.S. A direct approach to group decision making with uncertain additive linguistic preference relations. Fuzzy Optimization and Decision Making, 5(1):2132, 2006. 